\title{
The Role of Situation Awareness in Accidents of Large-scale Technological Systems
}

\author{
Mohsen Naderpour ${ }^{\mathrm{a}, 1}$, Salman Nazir ${ }^{\mathrm{b}, \mathrm{c}}$, Jie $\mathrm{Lu}^{\mathrm{a}}$ \\ ${ }^{\mathrm{a}}$ Decision Systems and e-Service Intelligence Laboratory, \\ Centre for Quantum Computation \& Intelligent Systems, Faculty of Engineering and IT, \\ University of Technology Sydney (UTS) \\ PO Box 123, Broadway, NSW 2007, Australia \\ ${ }^{\mathrm{b}}$ PSE-Lab-Dipartimento di Chimica, Materiali e Ingegneria Chimica, Politecnico di Milano, \\ Piazza Leonardo da Vinci 32, 20133 Milano, Italy \\ ${ }^{\mathrm{c}}$ Human Factors Research Group, Buskerud and Vestfold University College - Postboks 4, 3199 Borre, Norway
}

\begin{abstract}
:
In the last two decades, several serious accidents at large-scale technological systems that have had grave consequences, such as that at Bhopal, have primarily been attributed to human error. However, further investigations have revealed that humans are not the primary cause of these accidents, but have inherited the problems and difficulties of working with complex systems created by engineers. The operators have to comprehend malfunctions in real time, respond quickly, and make rapid decisions to return operational units to normal conditions, but under these circumstances, the mental workload of operators rises sharply, and a mental workload that is too high increases the rate of error. Therefore, cognivitive human features such as situation awareness (SA) - one of the most important prerequisite for decision-making - should be considered and analyzed appropriately. This paper applys the SA Error Taxonomy methodology to analyze the role of SA in three different accidents: (1) A runaway chemical reaction at Institute, West Virginia killing two employees, injuring eight people, and requiring the evacuation of more than 40,000 residents adjacent to the facility, (2) The ignition of a vapor cloud at Bellwood, Illinois that killed one person, injured two employees, and caused significant business interruption, and (3) An explosion at Ontario, California injuring four workers and caused extensive damage to the facility. In addition, the paper presents certain requirements for cognitive operator support system development and operator training under abnormal situations to promote operators' SA in the process industry.
\end{abstract}

Keywords: Situation awareness, Error taxonomy, Process safety, Abnormal situations, Operator support systems, Operators' training.

\footnotetext{
${ }^{1}$ Corresponding author, Tel: +61 295144520 .

E-mail addresses: Mohsen.Naderpour@uts.edu.au (M.Naderpour), Salman.Nazir@polimi.it (S. Nazir), Jie.Lu@uts.edu.au (J.Lu).
} 


\section{Introduction}

In the early morning hours of December $3^{\text {rd }}$ of 1984 , more than 40 metric tons of methyl isocyanate (MIC) gas leaked into the air from a pesticide plant located in the region of Bhopal, central India and caused one of the worst industrial disasters in history. Several hundred thousand people in towns nearby were exposed to the chemicals, and approximately 3,800 were killed immediately, at least 600,000 were injured, and at least 6,000 have died since (Broughton 2005). Three decades after the disaster, still high levels of contamination of toxic organic chemicals are found in the soil and water samples. The investigation of the disaster showed that on account of a series of mechanical and human errors in the production plant, water entered a tank containing a large amount of MIC, reacted exothermically and increased the temperature and pressure inside the tank, resulting in the release of MIC into the atmosphere. Although multiple factors including poor maintenance, the failure of safety systems and the substandard operating procedure have been identified as the underlying causes of the accident, the accident was officially blamed on human error as workers did not close the critical isolation valves before pipes were flushed with water and did not shut down the flare (Shrivastava, 1992).

The tragic event at Bhopal provides an extreme example of accidents in large-scale technological systems that have been attributed to human error. There are also several other accidents that show the difficulties of operators in working with complex systems or facing data overload. In fact, the majority of these accidents are caused by a combination of many factors which can be found in the lack of human factor considerations. Further investigation has revealed that of the human factors, operators' situation awareness (SA) is one of the most important prerequisite for decision-making (Endsley, 1995; Kaber and Endsley, 1998; Niu et al., 2009; Niu et al., 2013). Situation awareness describes how operators in dynamic complex systems develop and maintain a sufficient awareness of 'what is going on' in order to perform tasks successfully. Therefore, SA is likely to be at the root of many accidents in the process industry, where multiple goals must be pursued simultaneously, multiple tasks require the operator's attention, operator performance is under high time stress, and negative consequences associated with poor performance are anticipated (Naderpour et al., 2014b). In the case of the Texas City, TX BP Amoco Refinery explosion on 23 March 2005, where 15 workers were killed and 170 injured, several failures in control instrumentation and alarms caused an overfilled and over-pressurized tower to discharge a large quantity of flammable liquid into the atmosphere. The control room operator could not maintain good SA when monitoring this complex, dynamic environment, and an ignition created one of the worst industrial disasters in recent US history (Pridmore, 2007).

Today in many large-scale technological systems, operators are moved to a control room far away from the physical process, where automated systems pass more and more information to them. In fact, the automated systems and their over-deployment have changed the nature of operators work. In the past, the systems were analogue and a casual visit at the plant site was sufficient to monitor the progress and production of plants (Nazir et al., 2014c). Operators now must be alert in order to monitor, assess, and understand the incoming information from various sources and act/react accordingly. The decisions made by operators define the outcomes of possible abnormal situations, near misses, or even accidents. A recent 
report shows that the loss of abnormal situations cost 20 billion USD for US process plants every year. Among the attributes triggering these abnormal situations the contribution of human errors has been found to be 50\% (Walker et al., 2011).

This paper highlights the role of SA in three process accidents in recent US history taken from Chemical Safety Board (CSB) investigation reports (www.csb.gov), and presents certain requirements for improving operators' SA. The accidents include a runaway chemical reaction which occurred at a methomyl production facility, an explosion at an open top tank located in a chemical mixing area, and an explosion at an ethylene oxide sterilization facility. The accidents were formally investigated by CSB and directly blamed on human error; however, the role of SA remained unexplained which was intriguing for authors to investigate in this paper. The investigation reports provide sufficient real data, information and other material from these safety-critical environments than can help human factor analysts to conduct proper analyses. All of these are done in the following sections by (a) an introduction to SA and distributed SA in the process industry, (b) an accident analysis methodology, (c) three process accident analyses, (d) an overview of the requirements to maintain and promote SA in large-scale technological systems, and (e) concluding remarks.

\section{Situation Awareness and Process Industry}

To date, several SA models have been developed; however, Endsley's three-level model has undoubtedly received the most attention. This model describes SA as "the perception of the elements in the environment within a volume of time and space, the comprehension of their meaning and the projection of their status in the near future" (Endsley, 1995). The three-level model describes SA as an internally held product, comprising three hierarchical levels that is perception, comprehension, and projection, that is separate from the processes called situation assessment, used to achieve it. Operators actively try to construct a coherent, logical explanation to account for their observations. This cognitive activity involves two related concepts: the mental model and the situation model. Mental models refer to mechanisms whereby humans are able to generate descriptions of system purpose and explanations of system functioning. Mental models embody stored long-term knowledge about the systems that can be called upon during interaction with the relevant system when needed. A situation model is described as a schema depicting the current state of the mental model of the system. Endsley believes that the situation model provides a useful window on the broader mental model (Endsley, 2000b).

Kaber and Endsley (1998) believe that many of the performance and safety problems that currently occur in the process control arena are the result of difficulties with operators' SA. The analysis of offshore drilling accidents has revealed that more than $40 \%$ of such accidents are related to SA, and that the majority of those SA errors (67\%) occurred at the perceptual level, $20 \%$ concerned comprehension, and 13\% arose during projection (Sneddon et al., 2013). Nazir et al. (2012) highlight the importance and significance of SA for Field Operators and Control-Room Operators in the process sector and identify the major factors that influence their SA. Naderpour et al. (2014c) highlight the role of SA in performance of process operators when they confront abnormal situations and propose a method to model the operators'

mental models about such situations using Bayesian networks (Naderpour et al., 2015). They then 
developed a cognition-driven SA support system to assist operators in safety critical environments (Naderpour et al., 2014b).

Today, in the process industry the overall performance of systems depends on coordinated work among individuals that have responsibility for different subsets of goals, different access to data, and different situation perspectives. Therefore, there is a growing interest in understanding the cognitive and collaborative factors that enable such teams to work effectively (Roth et al., 2006). Thus, the concepts of team SA and shared SA are equally important in this regard. The degree to which every team member possesses SA on these elements for task performance is team SA (Kaber and Endsley, 1998). Thus, the success or failure of a team depends on the success or failure of each of its team members. In contrast, shared SA is defined as the degree to which team members possess the same SA on shared SA requirements (Endsley and Jones, 2001). Shared SA allows team members to efficiently coordinate work by enabling them to understand what is going on with the task, interpret what others are doing, and anticipate what will happen next. It enables team members to anticipate the information and support needs of other team members, resulting in reduced need for explicit communication and improved action coordination (Roth et al., 2006). However, Stanton et al. (2006) showed that because of complexity of current socio-technical systems and the increasing presence of teams, the concept of shared SA is not able to explain appropriately the interactions between agents (both human and non-human) in subsystems. Therefore, the concept of compatible SA has emerged. Rather than possess shared SA, the new distributed SA (DSA) based on compatible SA suggests that team members possess unique, but compatible, portions of awareness. The distributive nature of process plants, the importance and necessity of coordination and communication among various team members, located at different locations, the coexistence of technical and non-technical personnel within different units/sections of the plant call for a greater appreciation of DSA. Generally, operators are expected to monitor recurrently the dynamics of the process and to make timely correct decisions based on their mutual comprehension deduced from the available information that is changing dynamically. Team members experience a situation in different ways, as defined by their own personal experience, goals, roles, tasks, training, skills and so on. So whilst some of the information required by two different team members may be 'shared' in the sense that they both need to attend to it as part of their job, their resultant understanding and use of it is different. Ultimately, the picture developed by each team member is unique to themselves. Compatible awareness is therefore the phenomenon that holds distributed systems together (Salmon et al., 2009). The first effort to use the DSA in improving process safety has been conducted by Nazir et al. (2014c). They explain how the ultimate consequences of abnormal situations depend on the shared understanding, compatibility, and effective communication among operators. They also highlight the importance of a shared mental model and joint cognition to facilitate communication and the subsequently necessary actions.

\section{The Accident Analysis Methodology}

This study aims to analyze the role of SA behind three process accidents. The current section reviews the taxonomies of human error, and presents the methodology of this paper. 


\subsection{Taxonomies of Human Error}

The most applicable taxonomies to the context of human error are those taxonomies that are predominantly conceptual in nature. They focus on understanding the cognitive process involved in the production of human error rather than describing the observable characteristic of the error. Five popular conceptual human error taxonomies include:

- Situation Awareness Error Taxonomy (Endsley, 1999)

- Model of Internal Human Malfunction (Rasmussen, 1982)

- Model of Unsafe Acts (Reason, 1990)

- Information Processing Model (Wickens and Flach, 1988)

- Human Factors Analysis and Classification System (HFACS) (Shappel and Wiegmann, 2000)

These taxonomies represent a comprehensive view of the different types of human error that lead to unsafe acts. Among these taxonomies, the HFACS and the SA Error Taxonomy provide the greatest benefit in categorizing the context of human error (Leiden et al., 2001). The impetus for HFACS came from the absence of taxonomies of latent failures and unsafe acts within Reason's Swiss Cheese model, which limited its utility as an aviation accident analysis method. HFACS was subsequently developed based on an analysis of aviation accident reports and provides analysts with taxonomies of failure modes across the following four levels: unsafe acts; pre-conditions for unsafe acts; unsafe supervision; and organizational influences (Salmon et al., 2012). In addition, no single taxonomy of human error had been generally accepted by accident investigators and human factor researchers for addressing all causal factors. Hence, it was the goal for developing HFACS that it be utilized as a comprehensive framework to be used to identify human error. Although HFACS is comprehensive, it lists "loss of SA" as a single human causal condition under the second level. Given the research efforts to understand how and what is needed to improve SA, the single line item for "loss of SA" is too simplistic. Hence, the use of SA Error Taxonomy seems more appropriate for this study.

\subsection{Situation Awareness Error Taxonomy}

The taxonomy for classifying and describing errors in SA is based on the three-level model developed by Endsley (1995). The taxonomy incorporates factors effecting SA at each of its three levels. This methodology has been relied upon in several researches to investigate the causal factors underlying major accidents. For instance, Endsley (2000a) applied this taxonomy to analyze aircraft accidents in the US. Jones and Endsley (1996) used the taxonomy in a more extensive study of SA errors in aviation incidents involving pilots and air traffic controllers. It has also been utilized in analyzing maritime accidents (Grech et al., 2002). Table 1 describes the SA Error Taxonomy elements (Endsley, 1995) and provides descriptions for such elements in the process industry. 
Table 1: SA Error Taxonomy.

\begin{tabular}{l}
\hline Error Type $\quad$ Error Description \\
\hline Level 1: Failure to correctly perceive information
\end{tabular}

Data not available

Data are not available due to failure of the system design to present it or failure in the Distributed Control System (DCS) or other communication and control systems.

Data hard to discriminate or detect

Data are available; however, control room conditions such as inadequate lighting, noise, and obstructions blocking view, or design limitations such as poorly presented process equipment data in human-system interfaces (HSIs), or because of nature of data, prevent operators to detect or discriminate data.

Failure to monitor or observe data

Data are available, but are not scanned due to simple omission, attentional narrowing, distractions due to multi-tasking, or high workload.

Misperception of data

Data are misperceived due to influence of prior expectations or misunderstood due to task distraction.

Memory loss

Forgetting information which is due to disruptions in normal routine or startup operations, or high workload.

Level 2: Failure to correctly integrate or comprehend information

Lack of or poor mental model

Poor mental model does not enable the combining of information needed to meet goals. Primarily associated with automated systems.

Use of incorrect mental model

Interpretation of cues through an expected, but wrong, mental model of the system behavior leads to the incorrect assessment of the situation

Over-reliance on default values $\quad$ Routine expectations of the system are assumed even though conflicting information is available, but not accessed.

Other

Information is not properly integrated or comprehended due to working memory lapses or other undetermined cognitive reasons.

Level 3: Failure to project future actions or state of the system

Lack of or poor mental model

Information of current state is correctly understood, but projection of that state into the near future fails because of poor understanding of how to do so.

Over-projection of current trends

The current state is projected into the future correctly. However, it is projected further into the future than for which the data is realistically valid. This, combined with not updating the projections at appropriate intervals, can lead to incorrect plans for the future.

Other

Projection of current state into the future fails because it is a demanding task that in a multi-tasking environment is not always performed. This is possibly due to the lower priority it is given or due to limits in cognitive resources.

\begin{tabular}{ll}
\hline General & \\
\hline Failure to maintain multiple goals & $\begin{array}{l}\text { Failure to maintain multiple goals in memory degrades SA across all } \\
\text { three levels. }\end{array}$ \\
Executing habitual schema & $\begin{array}{l}\text { Performing task automatically can result in important system cues } \\
\text { being overlooked. }\end{array}$ \\
\hline
\end{tabular}

Note: DCS is a dedicated system used to control manufacturing processes; it is connected to sensors and actuators, and uses set point controls to control process variables. 


\subsection{Methodology}

The SA Error Taxonomy described above was used to examine the following accidents. The data source used to support the analyses was the CSB reports (CSB, 2006, 2007, 2011). Initially three human factor analysts met to discuss the accidents and available data, information, and conducted interviews by the CSB. At any level, an SA error was induced by problems with system design including needed information not available, poorly presented, ambiguous, or presented in the wrong format. In addition, at any level, the errors in information processing including memory or attention limitations, failure in pattern matching or mental projection were considered. Although the SA Error Taxonomy does not cover the errors related to the DSA, the analysts also investigated these errors in occurrence of the accidents. By gaining an understanding of why SA problems occurred in these case studies, a summary was ultimately provided that paved the way in providing some recommendations in Section 5 to prevent many of these problems.

\section{The Role of Situation Awareness in Process Accidents}

Loss of SA, poor SA and lack of SA as identified causal factors are now popular terms in accident investigation reports among several domains including aviation, nuclear industry, power plants, military, and process industry (Salmon and Stanton, 2013). Although, SA itself is not the only cause of accidents, it plays an important role in operators' decision making in time- and safety-critical situations (Naderpour et al., 2014a). This section applies the SA Error Taxonomy to analyze the accidents.

\subsection{The Explosion at Institute, West Virginia}

On 28 August 2008 a runaway chemical reaction occurred at a methomyl production facility in Institute, West Virginia, USA. Highly flammable solvent sprayed from a 4,500 gallon pressure vessel known as a residue treater and immediately ignited, killing two employees and injuring eight firefighters and contractors. The intense fire burned for more than four hours, more than 40,000 residents were evacuated to shelter-in-place for over three hours, and the highway was closed for hours because of smoke disruption to traffic (CSB, 2011). Figure 1 shows the facility damage and aerial view of reported damaged properties.

\subsubsection{Process Description}

Methomyl is a white, crystalline solid insecticide with a slight sulfurous odor that can form an explosive mixture when dispersed in air. Methyl isocyanate (MIC) is one of the key chemicals used to make methomyl. It is highly reactive with water and must be stored in stainless steel or glass containers at temperatures below $40^{\circ} \mathrm{C}$ to prevent a highly exothermic reaction. The methomyl production process begins by reacting aldoxime with chlorine to make chloroacetaldoxime, which reacts with sodium methyl mercaptide to produce methylthioacetaldoxime (MSAO). MSAO reacts with methyl isocyanate to produce methomyl. Excess MIC is removed from the methomyl-solvent solution and the solution is then pumped to the crystallizers where an anti-solvent is added to cause the methomyl to crystallize. Finally, the crystallized methomyl is separated from the solvents in the centrifuges and the methomyl cake is 
removed, dried, cooled, packaged in drums, and moved to the warehouse. The residual liquid from the centrifuges contains very small quantities of methomyl and other impurities (CSB, 2011).

Distillation separates the solvents in solvent recovery flashers and recycles the solvents to the start of the process. The unvaporized solvents and impurities, including up to 22 percent methomyl, accumulate in the bottom of the flasher. The flammable liquids can be used as fuel in the facility steam boilers, but before this flammable waste liquid can be pumped to an auxiliary fuel tank, the methomyl concentration has to be reduced to not more than 0.5 percent by weight for environmental and processing considerations (CSB, 2011).

The residue treater, which is a pressure vessel with a maximum allowable operating pressure of 50 psig, is used to dilute the incoming flasher bottoms, and is designed to operate at a sufficiently high temperature, and with sufficient residence time, to decompose the methomyl in the flasher bottoms stream to below 0.5 percent by weight. The solvent and residual waste material is transferred to the auxiliary fuel tank for use as a fuel in the facility steam boiler. Vapor generated in the methomyl decomposition reaction exits through the vent condenser to the process vent system where toxic and flammable vapor is removed (CSB, 2011).
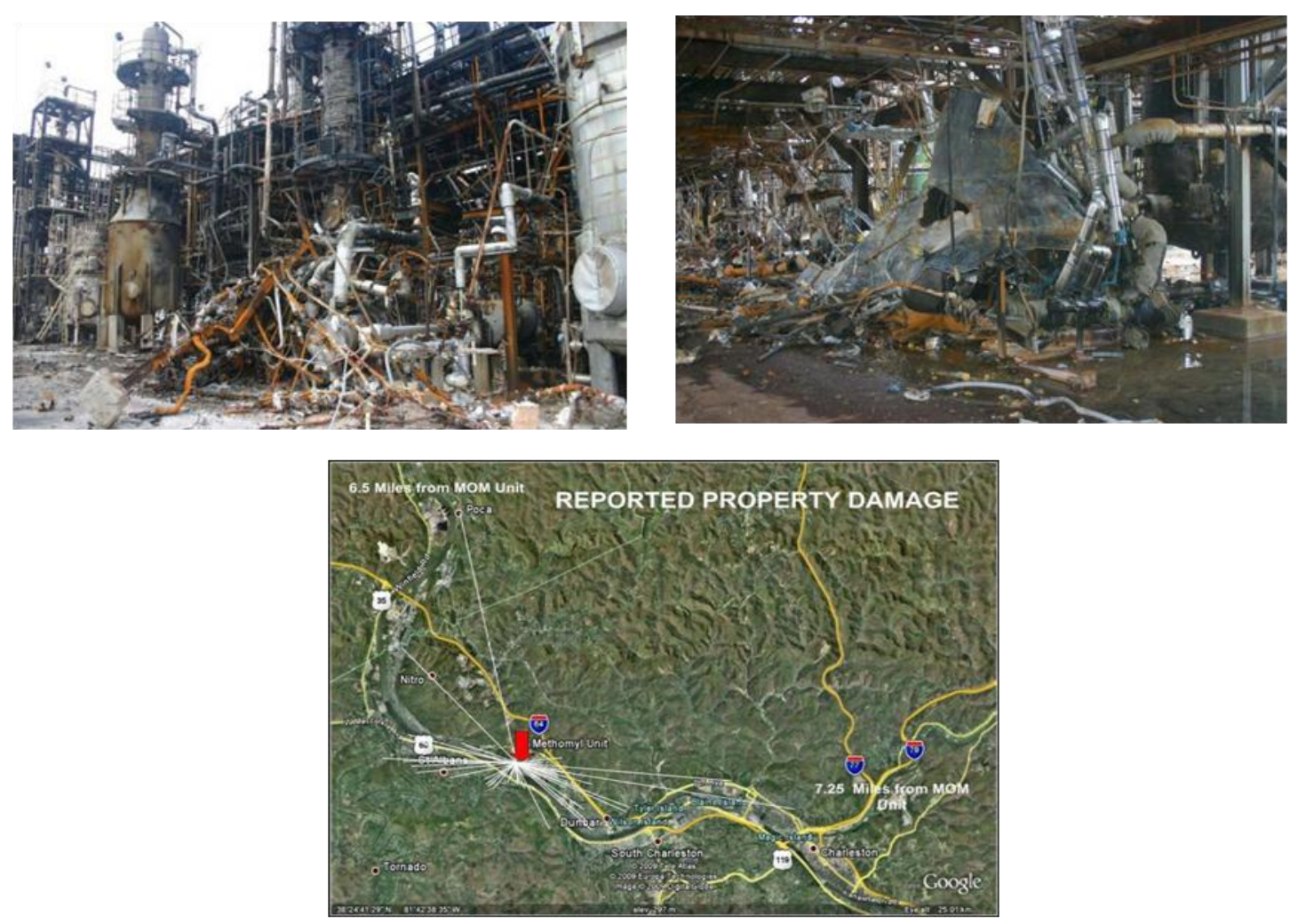

Figure 1: Facility damage and aerial view of reported damaged properties (CSB, 2011).

\subsubsection{Accident Timeline}

The residue treater accident occurred during the first methomyl restart after an extended outage to install a new process control system and a stainless steel pressure vessel. On the day of the accident at approximately 4:00, the outside operator manually opened the residue treater feed control valve and 
began feeding flasher bottoms into the almost empty vessel. With a low flow rate of about 1.5 gallons per minute, more than 24 hours would be required to fill the residue treater to 50 percent, the normal operating level. The outside operator started the recirculation pump at 18:15, as directed by the board operator. The residue treater liquid level was approximately 30 percent (1,300 gallons), the temperature ranged between $60^{\circ} \mathrm{C}$ and $65^{\circ} \mathrm{C}$, still significantly below the critical decomposition temperature of $135^{\circ} \mathrm{C}$, and the pressure remained constant at $22 \mathrm{psig}$. At 18:38, the temperature began to steadily rise at a rate of about 0.6 degrees per minute. At 22:21, the level was 51 percent when the recirculation flow suddenly dropped to zero. In less than three minutes, the temperature reached $141^{\circ} \mathrm{C}$, rapidly approaching the safe operating limit of $155^{\circ} \mathrm{C}$, and was climbing at the rate of more than two degrees per minute. At approximately 22:25, the residue treater high pressure alarm sounded at the work station. The board operator immediately observed that the residue treater pressure was above the maximum operating pressure and climbing rapidly but did not understand what was wrong. He therefore asked two outside operators to investigate why the pressure in the residue treater was unexpectedly increasing. About 10 minutes later, it suddenly and violently ruptured (CSB, 2011).

Approximately 2,200 gallons of flammable solvents and toxic insecticide residues sprayed onto the road and into the unit and immediately erupted in flames as severed electrical cables, or sparks from steel debris striking the concrete, ignited the solvent vapor. Debris was thrown in all directions, to a distance of some hundreds of feet. The blast over-pressure moderately damaged the unit control building and other nearby structures. Fortunately, a steel blanket protected a 6,700-gallon methyl isocyanate storage tank from flying debris and from the radiant heat generated by the nearby fires that burned for more than four hours. One employee died at the scene from blunt force trauma and thermal burn injuries, and the second employee died 41 days later. Residences, businesses, and vehicles as far as seven miles from the explosion epicenter sustained over-pressure damage that included minor structural and exterior damage, and broken windows. Acrid, dense smoke billowed from the fire into the calm night air for many hours. Smoke drifted over nearby roads, forcing many road closures and disrupting highway traffic. Methomyl and solvents were released from the residue treater, and solvents and other toxic chemicals, including flammable and toxic MIC, were released from ruptured unit piping. The released chemicals rapidly ignited, producing undetermined combustion products (CSB, 2011).

\subsubsection{Accident Analysis}

The runaway chemical reaction and loss of containment of the flammable and toxic chemicals was the result of deviation from the written start-up procedures and bypassing of critical safety devices intended to prevent such a condition occurring. In addition, it was indicated that inadequate DCS checkout and a poor human-system interface (HSI) prevented the operators from achieving correct operating conditions and adequate SA (Naderpour et al., 2014a).

The new DCS system included several display screens that were designed to mimic the process flow incorporated automated icons for critical equipment, and to show operating status and other parameters; Figure 2 shows the installed DCS interface. 


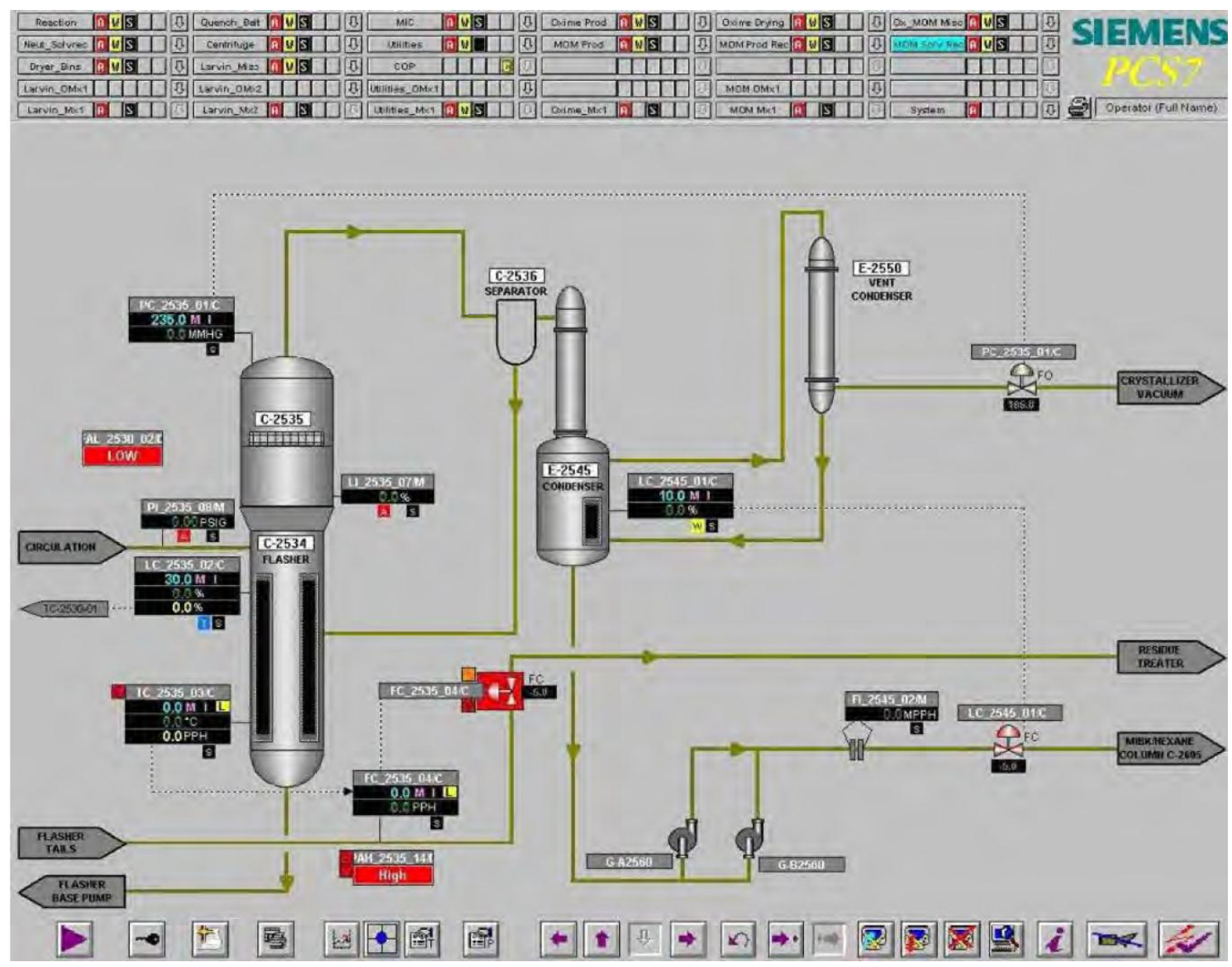

Figure 2: The DCS interface (CSB, 2011).

In this case, the data were available to the board operator and no loss of communication with the DCS or any failure in the DCS, were reported. However, the new control system significantly changed the interactions between the board operators and the DCS interface. The new visual displays and modified command entry method changing from a keyboard to a mouse influenced the usability of the HSI and impaired human performance. The increased complexities of the new operating system challenged operators as they had to familiarize themselves with the system and units of measurement for process variables that differed from those in the previous system. In addition, the new work station had five display screens available to monitor the processes and one display screen dedicated to process alarms. Some methomyl equipment required the operators to use at least three of the five display screens. The routine activities like starting a reaction or troubleshooting alarms would require operators to move between multiple screens to complete a task. Therefore, it concluded that the data were very hard to discriminate and deviation from safety set points to be detected.

In addition to identified level $1 \mathrm{SA}$ errors, four level $2 \mathrm{SA}$ errors that might have occurred were determined. Firstly, there was a lack of a good mental model, most frequently associated with the new automated system, as the facility management did not provide comprehensive formal training and practice using the new DCS for the operators. They incorrectly assumed the methomyl and oxime board operators had become proficient from the many operating hours using the DCS on the Larvin unit, which was 
adjacent unit to the residue treater. Secondly, it is worth noting that the wrong mental model or the mental model of a similar system, i.e. methomyl unit, might be used to interpret information, leading to an incorrect diagnosis or understanding of the situation. Thirdly, over-reliance on defaults in the mental models might be another problem. These defaults could be thought of as general expectations about how parts of the system function that might be used in the absence of real time data. Finally, it was also possible that several pieces of information were not properly integrated because of working memory limitations or other unknown cognitive lapses.

Apart from individual SA errors, another important contributing factor can be referred to inadequate SA among night shift and day shift operators. Night shift outside and board operators did not inform the day shift crew that they had started filling the residue treater with flasher bottoms, and the methomyl unit day shift operator neglected to inform the incoming night shift operator that the lab results from the scheduled flasher bottoms sample identified excessively high methomyl concentration. This can be attributed to loss of DSA as the lack of communication among the agents, which are different teams in this case, resulted/enabled the accident.

\subsection{The Explosion at Bellwood, Illinois}

On 14 June 2006, the ignition of a vapor cloud generated by mixing and heating a flammable liquid in an open top tank located in a chemical mixing area in Bellwood, Illinois, a suburb of Chicago, killed one contractor and injured two employees, and caused a significant business interruption. The accident occurred when an operator was mixing and heating a flammable mixture of heptane and mineral spirits in a 2,200-gallon tank equipped with steam coils (Figure 3). The finished product, "Super Clean and Tilt", is a proprietary mixture which is applied to cured concrete surfaces to prevent bonding with wet concrete (CSB, 2007).
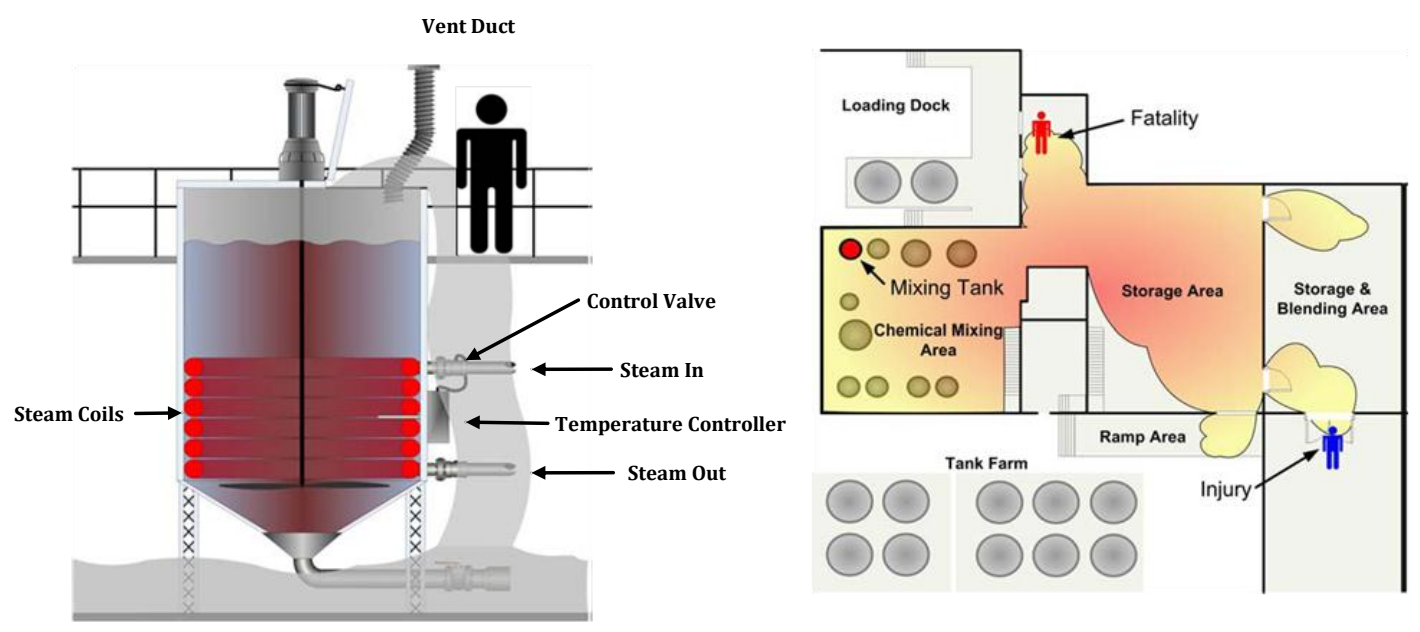

Figure 3: Vapor spilling and vapor cloud propagation (CSB, 2007).

\subsubsection{Process Description}

The process for making Super Clean and Tilt required several hours of mixing and heating. To begin heating, the operator manually opened the steam valves to the tank heating coils and adjusted the temperature controller to maintain the temperature at $73^{\circ} \mathrm{C}$. When the batch process was completed, the 
operator closed the steam valves and allowed the mixture to cool. The mixing tank was not equipped with a temperature display or high temperature alarm, and there was no backup shutoff device. The procedure for this mixture required the operator to verify the temperature by climbing the stairs to the upper level to measure it using a hand-held infrared thermometer, to monitor the situation and to conduct appropriate actions when necessary (CSB, 2007).

\subsubsection{Accident Timeline}

On the day of the accident, when the operator was adding an ingredient to the batch, he observed a "dense fog" accumulating on the floor below the tank. He immediately notified a senior operator who helped him shut down the operation. They both exited the building and advised workers in adjoining areas to leave. As the vapor cloud spread throughout the mixing area and surrounding workspaces, other employees exited the building. Within about 10 minutes after the operator first observed the vapor cloud, most employees who were working in the area had evacuated before the cloud got ignited. The pressure created by the ignition blew the doors open to an adjacent area, killing a contracted delivery driver and injuring two employees. The Bellwood Fire Department battled a fire confined to a bagged resin storage area for about three and one-half hours. The fire and pressure from the initial ignition produced moderate damage to the structure and interrupted operations for one month (CSB, 2007).

\subsubsection{Accident Analysis}

The most important contributing factor to the accident was associated with the physical environment, i.e. the temperature controller malfunctioned, which allowed the steam valve to remain open and heat the mixture to its boiling point. At the basic level, important information i.e. the inside of the tank temperature was not available to the operator, due to a failure of the system design. Furthermore, the system lack of a high temperature alarm, made it difficult for the operator to perceive important information which therefore contributed to the operator's reduced SA, resulting in the overflow of vapor from the tank. As the operator was responsible to verify the temperature during the production cycle, another hypothesis is that the information was available via infrared thermometer, but for various reasons, was not observed by the operator. This is due to several factors, including simple omission, attentional narrowing and external distractions that prevented the operator from attending to important information. High taskload, even momentary, might be another factor that prevents important information from being attended to. It is also probable that the operator attended to the temperature, but misperceived due to the influence of prior expectations, i.e. seeing what was expected rather that what was there. Finally, it was even possible that the operator initially perceived information then forgot about it due to high workload.

\subsection{The Explosion at Ontario, California}

On 19 August 2004, an explosion inside an air pollution control device and medical products sterilization chamber at an Ethylene Oxide (EO) sterilization facility in Ontario, California, injured four workers and severely damaged the facility (Figure 4). Neighboring businesses were evacuated for several hours and operations at the facility were disrupted for nine months (CSB, 2006). 

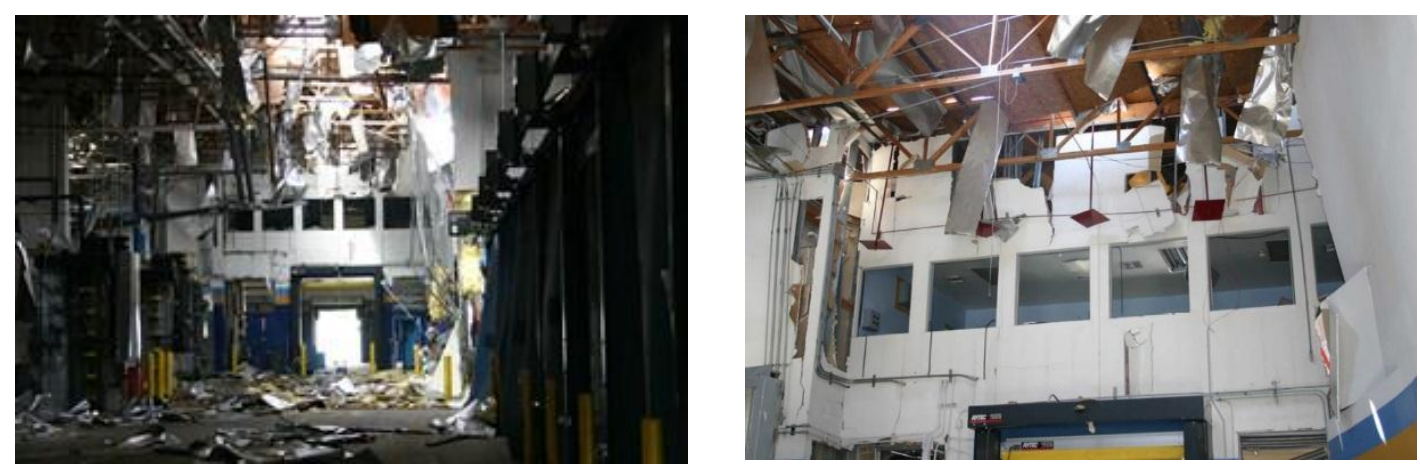

Figure 4: Facility damage (CSB, 2006).

\subsubsection{Process Description}

Ethylene Oxide presents an exposure hazard in addition to its high flammability. It kills microbes by disrupting life-sustaining molecules. Cycle variables include EO concentration, duration of exposure, temperature, humidity, vacuum applied during sterilization, and gas washing and aeration required to remove residual EO. Pre-conditioning is the first stage of the medical product sterilization process. It lasts from 6 to 24 hours and involves subjecting products to high levels of humidity, and temperatures between 27 and $49^{\circ} \mathrm{C}$. Operators use forklifts to move products to the sterilization chambers. The sterilization process begins by placing pallets of products inside a large stainless steel chamber, applying a vacuum, and injecting pure EO to achieve a sterilizing concentration of approximately 400,000 ppm. At the end of this phase, the chamber gas mixture is evacuated to the acid scrubber that removes EO. Despite efforts to remove all of the EO from sterilized products, potentially toxic levels of EO remain in the chamber after gas washing. To purge this remaining EO, operators open the sterilizer door to approximately six inches, which automatically opens a ventilation duct located in the rear of the chamber. Operators leave the door in this position for several minutes to ventilate the chamber so that employees can safely enter to remove sterilized products. Air exhausted through the back-vent flows to the oxidizer, which removes the remaining EO from the airstream. After ventilating the chamber, operators completely open the sterilizer door and use forklifts to move products to the aeration rooms. Circulating air in the aeration rooms, also vented to the oxidizer, removes any remaining residual EO (CSB, 2006).

The sterilization cycle is monitored and controlled from a computerized process control system located at the west end of the facility. The system automatically controls levels of humidity, temperature, pressure, EO, and dwell time. Facility management staff program cycle parameters and event sequencing into the system during the cycle design phase, based on specifications to achieve FDA ${ }^{1}$-mandated sterilization parameters. The system then controls the sequencing of that cycle from start to finish. Taking actions to manually intervene (advance or interrupt) a cycle sequence may present a considerable safety hazard because there is no monitoring or detection equipment to warn employees that an explosive concentration remains in the chamber (Nazir et al., 2014a). If an unrecoverable problem occurs during the sterilization cycle, operators can immediately abort the cycle by activating a button located on the control

\footnotetext{
${ }^{1}$ Food and Drug Administration
} 
room console. This initiates a pump that removes the high concentration gas from the sterilization chamber, followed by a sequence of gas washes that removes the remaining EO (CSB, 2006).

\subsubsection{Accident Timeline}

On the day of the accident, at approximately 1:30, the control system alerted operators of an EO injection failure during a cycle in Chamber 7. The operator immediately ran several routine system checks in the control room to determine that the alert was accurate, but was unable to identify any problems. The supervisor then decided to abort the cycle. In accordance with company protocol, they used the cycle abort button on the control room console. Upon completion of the abort cycle, operators removed the chamber contents to an aeration room, and the chamber was left open awaiting maintenance personnel. The maintenance supervisor arrived at the plant at approximately 7:30 and immediately assigned two technicians to work on the gas injection problem. He allowed maintenance personnel to enter a password to override computer safeguards, resulting in premature opening of the sterilizer door. Soon after that, the lower explosion limit alarm in the chamber was triggered, indicating the release of EO. The ignition of EO-air mixture took place before the oxidizer could be shut down (CSB, 2006).

\subsubsection{Accident Analysis}

A deeper look into the events reveals that the operators had difficulties in understanding the behavior and limitations of the automated system, relied upon poor mental models, which thus induced incorrect assumptions and led to wrong actions. The drawback of improperly designed automated systems was the progressive reduction of process understanding by the operators as they spent more time in passive vigilance instead of taking active decisions/actions. Consequently, they were unable to perform correctly when the system called for unconventional and even manual actions under abnormal situations.

The poor mental model or over projecting current trends also made it difficult for the operators to project the possible consequences of their decisions in the near future. In some cases, operators may be fully aware of what is going on, but be unable to correctly project what that means for the future. Generally, mental projection is a very demanding task at which people are poor. The lack of adequately designed job-specific maintenance-training methods was explicitly concluded by the analysts.

\subsection{Summary}

Overall, the accident analyses indicated that the primary cause of human errors in these environments was not related to poor decision making, but lack of SA. These errors were classified according to the SA Error Taxonomy. In the first accident, one of the identifiable SA errors was a level 1 SA error involving a failure to correctly discriminate data due to failures in appropriate design of a newly installed system and the related HSI. In addition, several level 2 SA errors due to lack of proper mental models have been identified. The second incident mainly resulted because of level 1 SA errors due to lack of appropriate operation system design. The occurrence of the third accident was attributed to levels 2 and 3 SA errors mainly due to poor mental models that prevented the operators from correctly comprehending the significance of the information and projecting the future state of the system. In summary, the identified SA errors can be categorized in two groups: 
(1) Errors due to lack of appropriate design of operator support systems and proper presentation of information in HSIs: traditionally, the focus of most human-system studies is on the technical elements, and human factors are often neglected. This is due to well understood hardware reliability techniques, whereas the handling of human factors, by contrast, is difficult. These problems highlight the urgent need to discover cognitive decision support systems to lower operator workload and stress and consequently reduce the rate of errors made by operators.

(2) Error due to poor mental models: as mental models refer to mechanisms whereby humans are able to generate descriptions of system purpose and form, explanations of system functioning and observed system states, and predictions of future states, the best way to change a mental model is through training. The training helps operators to describe the system, explain the system functioning and observed system states, and to predict the future system state, all of which are compatible with the three-level SA model. The Endsley approach presents mental models as default information that helps to form higher levels of SA even when needed data is missing or incomplete.

\section{Promoting Operators' Situation Awareness}

Promoting SA is now an important design objective for process systems where the information flow is high and distributed among various agents, and poor decisions may lead to accidents and disasters. In today's process systems which employ digital instrumentation, control systems and computer-based human-system interfaces (HSIs), operators need a greater level of support to control and maintain the facilities in safe conditions due to an increasing amounts of information that are passed to them via automated systems. In addition, the dynamics of normal operating conditions vary according to the state of the process. They may be in abnormal situations; therefore, different algorithms and actions to handle such situations should be considered during the design phase. The existing literature have several studies showing the improvement of performance based on good SA maintained by the operators (Burkolter and Kluge, 2012).

Traditionally, there are several approaches to prevent human error during operation of safety-critical systems; two important ones refer to the provision of better training programs for operators, and the improvement of operator support systems (Lee and Seong, 2014). The purpose of this section is to address these issues in the context of human factors and plant safety performance. Firstly, some requirements for developing cognitive operator support systems that can assist operators in their decisionmaking from a human cognition perspective are presented. Secondly, a new operator training approach based on a real-time dynamic process simulator, a real time dynamic accident simulator amalgamated with immersive environment is introduced. The recommendations would promote operators' SA during normal and abnormal situations and consequently reduce human error and improve process systems performance.

\subsection{Operator Support Systems}


Chemical processes contain multilevel control loops and interconnections, which need to be monitored and supervised for normal operations. Once the system becomes unstable, the conditions are referred to as abnormal situation, which can lead to near misses and possible accidents with both economic and human loss. The process plants have experienced a significant increase in multidimensional automation in the last two decades that have significantly increased the complexity and sensitivity of the role of operators and their teams. However, they lack the ability to intervene or tackle abnormal situations as they are usually designed for routine operating conditions (Nazir et al, 2014a). Therefore, any attempt to develop operator support systems should consider both normal and abnormal situations. Most prior support systems focus on the deviation of the process from an acceptable range of operation. Therefore, the use of quantitative knowledge and hardware failures has been relied on significantly. Most of them consider the identification of operation faults (Qian et al., 2008) or the prediction of process variables (Juricek et al., 2001) that will violate an emergency limit in the future; however, further research show that when faults occur, operators have to rely on their experience under working pressure to understand what is going on and to contribute a solution (Kluge et al., 2014). Therefore, designing and integrating appropriate approaches to develop cognitive support systems are highly recommended (Naderpour et al., 2014b; Niu et al., 2013).

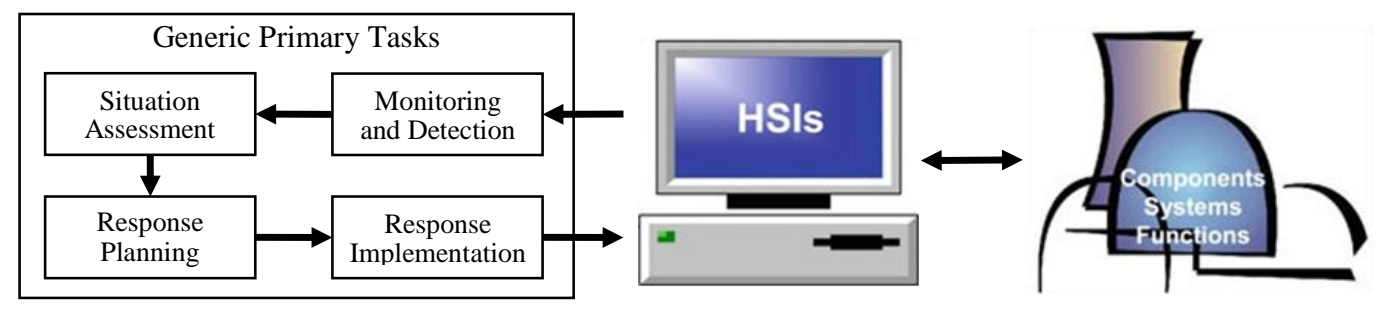

Figure 5: General primary tasks.

Generally, operators perform two types of tasks to carry out their roles and responsibilities: primary tasks and secondary tasks. As illustrated in Figure 5, primary tasks consist of several cognitive tasks including monitoring and detection, situation assessment, response planning, and response implementation (O’Hara and Persensky, 2011). Any breakdown in generic primary tasks can lead to a human error. Therefore, a balanced automated system that avoids an excessive workload for the operators and keeps them in the loop of decision-making, taking action, and updating the related information would benefit the process industry. The activities involved in extracting information from the environment are referred to as monitoring and detection. In today's process systems, these tasks are highly supported through various heterogeneous sensors and appropriate signal-processing methods that are used to extract as much information as possible about the dynamic environment. Good monitoring results in operator's perception or SA level 1.

Situation assessment is the evaluation of current conditions to determine that they are acceptable or to determine the underlying causes of abnormalities. Situation assessment which underlies the achievement of SA is therefore critical to taking proper human action. Thus, the HSI besides providing alarms and displays that are used to obtain information to support situation assessment must provide additional 
support for assessing a situation. This development corresponds to SA levels 2 and 3 that support operators to infer real situations and to project their status in the near future. Response planning refers to deciding upon a course of action to address the current situation. In general, response planning involves operators using their situation model to identify goal states and the transformations required to achieve them. Response implementation means performing the actions specified by response planning. These actions include selecting a control, providing control input, and monitoring the system and process response (O’Hara and Persensky, 2011).

Apart from primary tasks, operators perform another kind of tasks that are referred to secondary tasks or "interface management tasks" such as navigating, configuring and arranging, that assist operators to perform the primary tasks successfully. Secondary tasks create workload and may take so much attention away from primary task performance and generate a "keyhole effect" (Seong, 2009) and thus affect operators' SA, which takes operator out-of-the-loop. Thus, secondary tasks should be carefully addressed in design reviews as well. In actual plant operation, individual operators typically do not perform these tasks alone; tasks are accomplished by the coordinated activity of multi-person teams. Therefore, the design of technology needs to consider not only individual performance but also team performance.

\subsection{Operators Training}

Inclusion of automation, advancement in technology, and sophisticated tools/software in the process industry have changed the roles and goals of the industrial operators. Therefore novel training paradigms are needed to couple to a cross-disciplinary approach, which can facilitate the operators performing their tasks better thus ensuring smoother, safer, and optimal operations within the available degrees of freedom. Even though the significance of training and skills development have been held in high esteem in domains like aviation and the military, limited resources have been allocated to this in the case of the process industry. Unfortunately, the lack of cross disciplinary expertise for the development and implementation of training methods has kept this domain in the 'too hard basket'.

We propose the use of immersivity during the training of field as well as control room operators (Nazir et al. 2013). The immersive nature is essential for increasing the reliability of the training experience and for producing a positive impact on the psychological experience of the operator. "Immersion" is the subjective impression that one is participating in a holistic and realistic experience. The training tool proposed by the authors is a Plant Simulator (PS), which is capable of linking to any conventional Operator Training Simulator (OTS) and of exchanging information through a two-way communication channel. By incorporating in the OTS, the PS allows running the simulation of conventional operations, abnormal situations, and even accident events. The process simulator, accident simulator, 3D engine and performance assessment algorithms work in the background and show their results by means of the virtual immersive environment (Figure 6). 


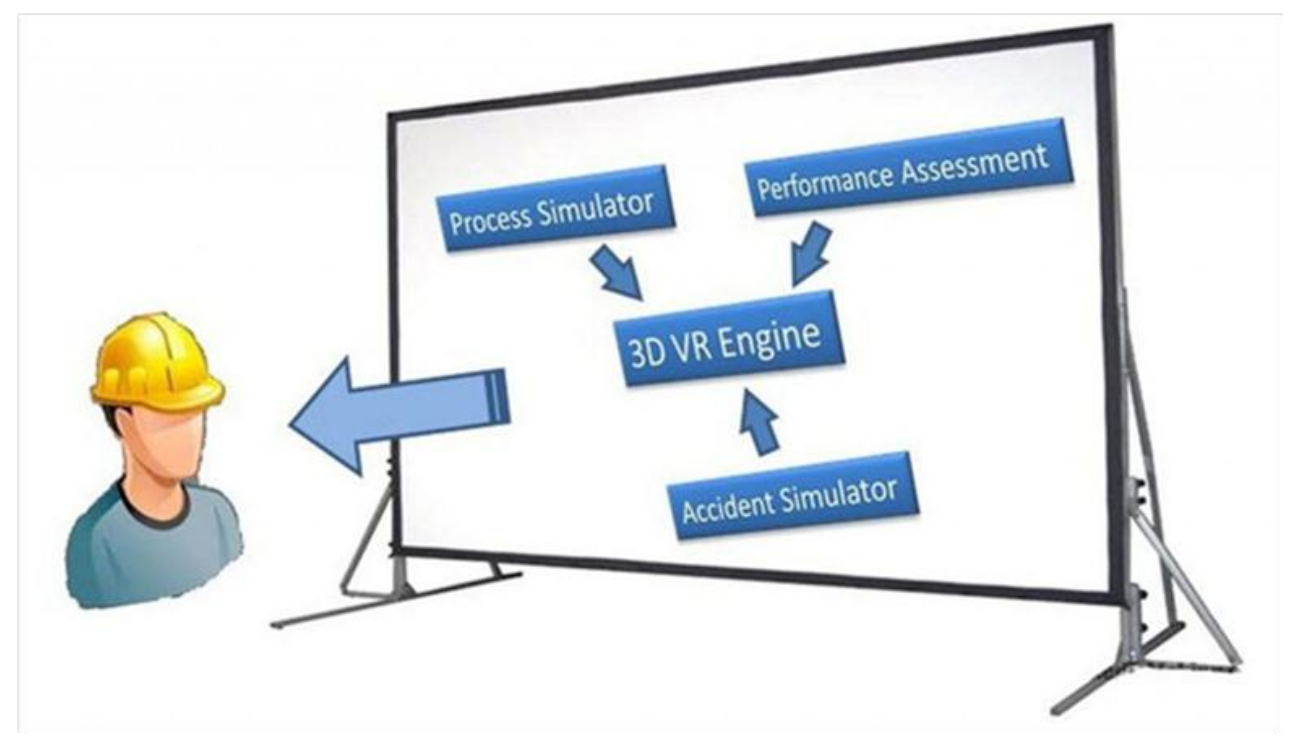

Figure 6: A sketch of the Plant Simulator (PS) environment where the operator faces the 3D screen during the training session.

The PS training not only supports mental mapping and retention abilities of the participants, but also sets up an effective communication among operators working in different plant sections. It allows the operator to learn, understand, and practice the process details, control loops, interconnections, and constraints in a 3D immersive environment. In addition, simulated automation failures (which cannot be tested in a real plant) instill the skills of handling malfunctions, abnormal situations, coordination, skill acquisition and adaptability. This unique training method integrates the details of process plants, interconnections, and control loops and allows the operators to experience real situations (in a virtual environment) by practicing the tasks and experiencing the imperfect features of automated systems and their possible consequences. Adequately trained operators can properly interact with automated systems so to transform automation from curse to cure (Nazir et al. 2014a).

For practice-based training, feedback on results as well as on performance is vital in order to correct the operators' mental model and to infer objectives for improvement. Therefore, the PS incorporates a complete automated assessment of the operator performance, which can allow the operators and managers to track the learning abilities during training sessions. Further details about the necessity of automated performance assessment and the algorithms that allow this novel methodology can be found in Manca et al. (2014). The PS has also demonstrated its efficacy in training operators with respect to well-established procedures that have existed since last two decades. For instance, polymerization processes are widespread and often based on catalytic reactions (Urdampilleta et al., 2006). Frequently, the addition of catalyst to the continuous operation is performed through injectors, which allows the catalyst to be mixed to the reacting flow. Such a procedure is run periodically (for example every month) and requires switching the catalyst inlet stream between the working and the spare injectors. The procedure requires several valves and buttons to be operated and pressure, temperature, and flow rate gauges to be read, while waiting for some process lines to be deactivated and finally reactivated. The overall procedure can 
take a few minutes, but even a small error in the operation sequence may have severe repercussions for the plant. A single operation run out of sequence or at the wrong time may cause the shutdown of the whole plant. The loss of production is then dramatic and may reach millions of dollars in lost revenues. Again, the PS tailored for the section of the plant where the catalyst switch procedure is performed periodically, allows the operator to train and test his/her preparation with respect to the assigned sequence of actions (for example see Figure 7).

The possibility of performing the simulated procedure several times, in front of the virtual reproduction of the plant, increases the self-efficacy of the trainee, who can measure his/her degree of training through the automated performance assessment. By practicing the training session several times, the trainee can track his/her improvements. This experience also induces proceduralization of actions, thus relieving a proportion of cognitive load and psychological stress related to the criticality of the procedure. Our results shows that the awareness of the operators is significantly improved when training with PS compared to the conventional methodologies adopted to train operators (Nazir et al. 2013). During a simulated accident scenario, the participants training with PS were able to mitigate the impact of the accident while they were not able to do this when using conventional training methods.

As the tasks in the process industry requires collaborative efforts among team members, therefore, the 3D simulator also allows the operators (and even non-technical staff) to experience a stressful situation and resolve it by working together in exactly the same manner as they would be on the real plant. A simulated accident scenario can provide the teams with real training to communicate the abnormality, weigh and analyze the parameters, and developed a shared mental model to reach correct and timely decisions to avert the impact of the simulated accident. Specifically, experiencing an accident scenario can scaffold trainees in a systematic way to make decisions and observe the outcomes of their decisions with reference to the accident scenario and therefore, reach conclusions and learn lessons that can be implemented or capitalized on in a real abnormal situation at the plant.

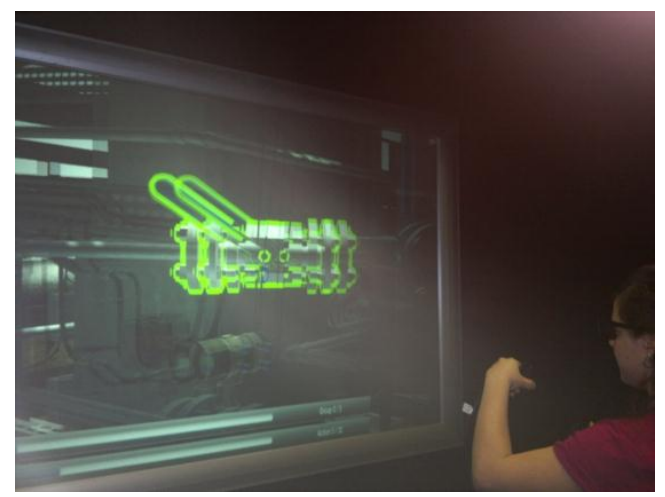

Figure 7: A trainee involved in the training session in the Plant Simulator catalytic reactions of polymerization processes (Nazir et al., 2014b). The distortion of the graphic of the Plant Simulator (green valve) is on account of its 3D nature that can be experienced with 3D glasses only, as worn by the trainee.

\section{Concluding Remarks}


Many attempts were made over the past 20 years to reduce human error in the process industry. The main conclusion is that few errors represent random events; instead, most human errors can be explained by human cognitive mechanisms. Of these cognitive mechanisms, an operator's situation awareness is considered to be one of the most important pre-requisite for decision-making, especially in time-, safetycritical abnormal situations. This paper reviewed the role of SA in three accidents in the process sector and analyzed the SA related errors based on SA Error Taxonomy methodology. The identified SA errors were able to be totally categorized in two groups; errors due to a lack of appropriate design of operator support systems, and errors due to poor mental models. Therefore, the urgent need to discover cognitive support systems in order to lower operator workload and stress and consequently human errors were highlighted. In addition, a novel operator training method using a Plant Simulator and an Immersive Virtual Environment was presented. More empirical studies aiming at determining the long-term benefits of operator support systems and advanced operator training simulators on operators' SA can deepen the insights offered here.

\section{Acknowledgment}

The work presented in this paper was supported by the Australian Research Council (ARC) under Discovery Project DP140101366.

\section{References}

Broughton, E., 2005. The Bhopal disaster and its aftermath: A review. Environmental Health: A Global Access Science Source 4, 6.

Burkolter, D., Kluge, A., 2012. Process control and risky decision-making: Moderation by general mental ability and need for cognition. Ergonomics 55, 1285-1297.

Chemical Safety Board, 2006. Sterigenics, Washington, DC.

Chemical Safety Board, 2007. Mixing and heating a flammable liquid in an open top tank, Washington, DC.

Chemical Safety Board, 2011. Pesticide Chemical Runaway Reaction Pressure Vessel Explosion, Washington, DC.

Endsley, M.R., 1995. Toward a theory of situation awareness in dynamic systems. Human Factors: The Journal of the Human Factors and Ergonomics Society 37, 32-64.

Endsley, M.R., 1999. Situation awareness and human error: Designing to support human performance, Proceedings of the High Consequence Systems Surety Conference.

Endsley, M., 2000a. Errors in situation assessment: Implications for system design, in: Elzer, P.F., Kluwe, R.H., Boussoffara, B. (Eds.), Human error and system design and management. Springer London, pp. 15-26.

Endsley, M.R., 2000b. Situation models: An avenue to the modeling of mental models, Proceedings of the Human Factors and Ergonomics Society Annual Meeting. SAGE Publications, pp. 61-64.

Endsley M. R., Jones W. M. (2001). A model of inter- and intrateam situation awareness: Implications for design, training and measurement. In McNeese M., Salas E., Endsley M. R. (Eds.), New trends in cooperative activities: Understanding system dynamics in complex environments (pp. 46-67). Santa Monica, CA.

Grech, M.R., Horberry, T., Smith, A., 2002. Human error in maritime operations: Analyses of accident reports using the Leximancer tool. Proceedings of the Human Factors and Ergonomics Society Annual Meeting, pp. 1718-1721.

Jones, D.G., Endsley, M.R., 1996. Sources of situation awareness errors in aviation. Aviation, Space, and Environmental Medicine 67, 507-512.

Juricek, B.C., Seborg, D.E., Larimore, W.E., 2001. Predictive monitoring for abnormal situation management. Journal of Process Control 11, 111-128.

Kaber, D.B., Endsley, M.R., 1998. Team situation awareness for process control safety and performance. Process Safety Progress 17, 43-48.

Kluge, A., Nazir, S., Manca, D., 2014. Advanced applications in process control and training needs of field and control room operators. IIE Transactions on Occupational Ergonomics and Human Factors, (in press). 
Lee, S., Seong, P., 2014. Design of an integrated operator support system for advanced NPP MCRs: Issues and perspectives. In: Yoshikawa, H., Zhang, Z. (Eds.), Progress of Nuclear Safety for Symbiosis and Sustainability. Springer-Verlag, Tokyo, pp. 11-26.

Leiden, K., Keller, J., French, J., 2001. Context of human error in commercial aviation. Micro Analysis \& Design, Inc.

Manca, D., Nazira, S., Colomboa, S., Klugeb, A., 2014. Procedure for automated assessment of industrial operators. Chemical Engineering 36, 391-396.

Naderpour, M., Lu, J., Zhang, G., 2014a. The explosion at Institute: Modeling and analyzing the situation awareness factor. Accident Analysis \& Prevention 73, 209-224.

Naderpour, M., Lu, J., Zhang, G., 2014b. An intelligent situation awareness support system for safety-critical environments. Decision Support Systems 59, 325-340.

Naderpour, M., Lu, J., Zhang, G., 2014c. A situation risk awareness approach for process systems safety. Safety Science 64, 173-189.

Naderpour, M., Lu, J., Zhang, G., 2015. An abnormal situation modeling method to assist operators in safety-critical systems. Reliability Engineering \& System Safety 133, 33-47.

Nazir, S., Colombo, S., Manca, D., 2012. The Role of Situation Awareness for the operators of process industry. Chemical Engineering Transactions 26, 303-308.

Nazir, S., Colombo, S., Manca, D., 2013. Testing and analyzing different training methods for industrial operators: an experimental approach, in: Andrzej, K., Ilkka, T. (Eds.), Computer Aided Chemical Engineering. Elsevier, pp. 667672.

Nazir, S., Kluge, A., Manca, D., 2014a. Automation in process industry: Cure or curse? How can training improve operators' performance, in: Jiří Jaromír Klemeš, P.S.V., Peng Yen, L. (Eds.), Computer Aided Chemical Engineering. Elsevier, pp. 889-894.

Nazir, S., Kluge, A., Manca, D., 2014b. Can immersive virtual environments make the difference in training industrial operators?, Proceedings of the Human Factors and Ergonomics Society Europe Chapter 2013 Annual Conference, Turin, Italy, pp. 251 - 265.

Nazir, S., Sorensenb, L.J., Øvergårdb, K.I., Manca, D., 2014c. How distributed situation awareness influences process safety. Chemical Engineering Transactions 36, 409-414.

Niu, L., Lu, J., Zhang, G., 2009. Cognition-Driven Decision Support for Business Intelligence: Models, Techniques, Systems and Applications. Springer-Verlag, Berlin Heidelberg.

Niu, L., Lu, J., Zhang, G., Wu, D., 2013. FACETS: A cognitive business intelligence system. Information Systems $38,835-862$.

O'Hara, J.M., Persensky, J., 2011. Human Performance and Plant Safety Performance. In Britt Skjerve A. and Bye A. (Eds.), Simulator-Based Human Factors Studies Across 25 Years. Springer, pp. 91-106.

Pridmore, J.L., 2007. Designing for the improvement of operator situation awareness in automation systems. PhD thesis, Auburn University, Alabama, U.S.

Qian, Y., Xu, L., Li, X., Lin, L., Kraslawski, A., 2008. LUBRES: An expert system development and implementation for real-time fault diagnosis of a lubricating oil refining process. Expert Systems with Applications 35, 1252-1266.

Roth, E.M., Multer, J., Raslear, T., 2006. Shared situation awareness as a contributor to high reliability performance in railroad operations. Organization Studies 27, 967-987.

Rasmussen, J., 1982. Human errors. A taxonomy for describing human malfunction in industrial installations. Journal of Occupational Accidents 4, 311-333.

Reason, J., 1990. Human error. Cambridge University Press.

Salmon, P.M., Stanton, N.A., Walker, G.H., Jenkins, D.P., Rafferty, L., 2009. Is it really better to share? Distributed situation awareness and its implications for collaborative system design. Theoretical Issues in Ergonomics Science $11,58-83$.

Salmon, P.M., Cornelissen, M., Trotter, M.J., 2012. Systems-based accident analysis methods: A comparison of Accimap, HFACS, and STAMP. Safety Science 50, 1158-1170.

Salmon, P.M., Stanton, N.A., 2013. Situation awareness and safety: Contribution or confusion? Situation awareness and safety editorial. Safety Science 56, 1-5.

Seong, P.H., 2009. Reliability and risk issues in large scale safety-critical digital control systems. Springer-Verlag.

Shappel, S.A., Wiegmann, D.A., 2000. The human factors analysis and classification system-HFACS. US Federal Aviation Administration, Office of Aviation Medicine.

Shrivastava, P., 1992. Bhopal: Anatomy of a crisis. P. Chapman Pub, Ballinger, Cambridge, MA.

Smith, K., Hancock, P., 1995. Situation awareness is adaptive, externally directed consciousness. Human Factors: The Journal of the Human Factors and Ergonomics Society 37, 137-148. 
Sneddon, A., Mearns, K., Flin, R., 2013. Stress, fatigue, situation awareness and safety in offshore drilling crews. Safety Science 56, 80-88.

Stanton, N.A., Stewart, R., Harris, D., Houghton, R.J., Baber, C., McMaster, R., Salmon, P., Hoyle, G., Walker, G., Young, M.S., 2006. Distributed situation awareness in dynamic systems: theoretical development and application of an ergonomics methodology. Ergonomics 49, 1288-1311.

Sugeno, M., 1985. Industrial applications of fuzzy control. Elsevier Science Inc.

Urdampilleta, I., González, A., Iruin, J.J., de la Cal, J.C., Asua, J.M., 2006. Origins of product heterogeneity in the spheripol high impact polypropylene process. Industrial \& engineering chemistry research 45, 4178-4187.

Walker, P.D., Cammy, N.E., Ellis, B.J., Seibert, K.D., 2011. Operations skills for the 21st century, National Petrochemical \& Refiners Association (NPRA) Annual Meeting, Paper AM-11-68.

Wickens, C., Flach, J.M., 1988. Information processing. Human factors in aviation 86, 111-155. 